\title{
EL USO DE LAS TECNOLOGÍAS DE LA INFORMACIÓN Y LA COMUNICACIÓN EN EL APRENDIZAJE DE LA QUÍMICA
}

\author{
AUTORES: $\quad$ Yoiler Batista Garcet ${ }^{1}$ \\ Rolando Barcia Menéndez ${ }^{2}$ \\ Raquel Vera Velàzquez ${ }^{3}$ \\ Alfredo Lesvel Castro Landìn ${ }^{4}$ \\ Pedro Roberto Valdés Tamayo ${ }^{5}$
}

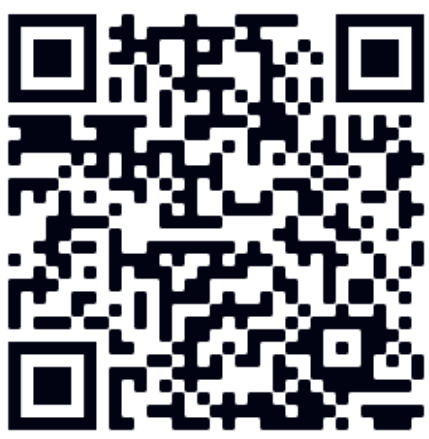

DIRECCIÓN PARA CORRESPONDENCIA:yoiler.batista@ unesum.edu.ec

Fecha de recepción: 13/02/2019

Fecha de aceptación: 08/04/2019

\section{RESUMEN}

La investigación realizada se centra en la preparación de los profesores de la facultad de Ciencias de la salud en el uso de las TICs para brindar las posibilidades didácticas en la adquisición de conocimientos teóricos y prácticos de la química, aprovechando el vertiginoso desarrollo de las tecnologías en el siglo XXI. Durante el proceso investigativo se ha utilizado, fundamentalmente, como método del nivel empírico, la observación, de carácter participante y sistemático, del proceso pedagógico que se lleva a cabo en la facultad. Para ello se ha elaborado como instrumento una guía de observación que ha permitido recolectar información sobre el empleo de las nuevas tecnologías en el desarrollo de las clases de química, lo que ha revelado la existencia del problema que se investiga. La observación ha posibilitado valorar la pertinencia de la propuesta que se presenta como solución al problema. Dicha propuesta consiste en brindar herramientas digitales y metodológicas que permita a los docentes explotar las potencialidades de dichos medios.Las TICs han tenido un gran impacto en el aprendizaje de la química en la facultad de Ciencias de la Salud de la Universidad Estatal del Sur de Manabí, para dar respuesta a las necesidades de los estudiantes en el logro de habilidades de formulación y experimentación. Este artículo científico se centra en la preparación de los profesores de la facultad de Ciencias de la salud en el uso de las TICs para

\footnotetext{
${ }^{1}$ la Salud, Universidad Estatal del Sur de Manabí, Campus Los Ángeles, vía Noboa Km 1 1⁄2 s/n, en Jipijapa, Manabí.

${ }^{2}$ M.Sc. Docente investigador de la Asignatura de Bioseguridad, Carrera de Laboratorio Clínico, Facultad de Ciencias de la Salud, Universidad Estatal del Sur de Manabí, Campus Los Ángeles, vía Noboa Km 1 1⁄2 s/n, en Jipijapa, Manabí. ${ }^{3}$ MSc. Docente investigador de la Asignatura de Matemáticas, Carrera Agropecuaria Facultad de Ciencias Naturales y de la Agricultura, Universidad Estatal del Sur de Manabí, Campus Los Ángeles, vía Noboa Km 1 1⁄2 s/n, en Jipijapa, ${ }^{4}$ MSc. Docente investigador de Asignatura de Química, Carrera Agropecuaria, Facultad de Ciencias Naturales y de la Agricultura, Universidad Estatal del Sur de Manabí, Campus Los Ángeles, vía Noboa Km 1 1⁄2 s/n, en Jipijapa, Manabí.

${ }^{5} \mathrm{PhD}$. Docente investigador de la Asignatura de Matemáticas, Carrera Agropecuaria, Facultad de Ciencias Naturales y de la Agricultura, Universidad Estatal del Sur de Manabí, Campus Los Ángeles, vía Noboa Km 1 1⁄2 s/n, en Jipijapa, Manabí.
} 
Yoiler Batista Garcet, Rolando Barcia Menéndez, Raquel Vera Velàzquez...

brindar las posibilidades didácticas en la adquisición de conocimientos teóricos y prácticos de la química.

PALABRAS CLAVE: Tecnología de la Información, experimentación, química, aprendizaje.

THE USE OF INFORMATION AND COMMUNICATION TECHNOLOGIES IN THE LEARNING OF CHEMISTRY IN STUDENTS OF THE CLINICAL LABORATORY CAREER OF THE SOUTHERN STATE UNIVERSITY OF MANABÍ, ECUADOR.

\begin{abstract}
The research carried out focuses on the preparation of professors of the Faculty of Health Sciences in the use of ICTs to provide the didactic possibilities in the acquisition of theoretical and practical knowledge of chemistry, taking advantage of the vertiginous development of technologies in The 21st century. During the research process has been used, primarily as a method of the empirical level, the observation, participatory and systematic, the pedagogical process that takes place in the faculty. To this end, an observation guide has been developed as an instrument that has allowed collecting information on the use of new technologies in the development of chemistry classes, which has revealed the existence of the problem being investigated. Observation has made it possible to assess the relevance of the proposal presented as a solution to the problem. This proposal consists of providing digital and methodological tools that allow teachers to exploit the potential of such media. ICTs have had a great impact on the learning of chemistry in the Faculty of Health Sciences of the Southern State University of Manabí, to respond to the needs of students in the achievement of formulation and experimentation skills. This scientific article focuses on the preparation of professors of the faculty of Health Sciences in the use of ICTs to provide the didactic possibilities in the acquisition of theoretical and practical knowledge of chemistry.
\end{abstract}

KEYWORDS: Information technology; experimentation; chemistry; learning.

\title{
INTRODUCCIÓN
}

\section{Las Tecnologías de la Información y la Comunicación}

Las Tecnologías de la Información y la Comunicación: "Son un conjunto de aparatos electrónicos, redes y servicios que se integran o interrelacionan, en un sistema de información interconectado y complementario. Son las técnicas de la computación asociadas a las de la Comunicación, utilizadas con diferentes fines". (Gónzález,E, 2019)

"En líneas generales podríamos decir que las nuevas tecnologías de la información y comunicación son las que giran en torno a tres medios básicos: la informática, la microelectrónica y las telecomunicaciones; pero giran, no sólo de forma aislada, sino lo que es más significativo de manera interactiva e interconexionadas, lo que permite conseguir nuevas realidades comunicativas". (Cardozo, J.C.G, Navarro, S.M.B \& Álvarez, D.J:S, 2017)

Para Antonio Bartolomé "la T.E. encuentra su papel como una especialización dentro del ámbito de la Didáctica y de otras ciencias aplicadas de la Educación, refiriéndose especialmente al diseño, desarrollo y aplicación de recursos en procesos educativos, no únicamente en los procesos instructivos, sino también en aspectos relacionados con la Educación Social y otros campos educativos. Estos recursos se refieren, en general, especialmente a los recursos de carácter 
informático, audiovisual, tecnológicos, del tratamiento de la información y los que facilitan la comunicación" (García-Valcárcel Muñoz - Repiso, 2017)

Diferentes estudios evidencian que las nuevas tecnologías pueden ayudar a los estudiantes a aprender y a los profesores a enseñar de un modo más efectivo. Las investigaciones sugieren que, aunque las Tecnologías de la Información y las Comunicación pueden mejorar el aprendizaje, hay un gran número de aspectos que necesitan ser considerados si queremos que las nuevas tecnologías conciban una diferencia efectiva. Por tanto, se requiere cierta cautela sobre dónde y cómo las mismas pueden tener un mayor impacto en el aprendizaje de la química.

Se han evidenciado beneficios en el uso de las Tecnologías y las comunicaciones en función del aprendizaje de la química entre estos podemos mencionar;

El uso de las herramientas de google como los formularios y el classrom les permite acceder rápido a la información y conocer los resultados de evaluaciones en un menor tiempo posible.

La nomenclatura química es el contenido que más difícil se les hace a los estudiantes y mediante el uso de la computadora y simuladores virtuales los mismos fijan de una manera mejor el contenido.

\section{DESARROLLO}

\section{2- El proceso de enseñanza}

La enseñanza, en cualquier asignatura es una acción coordinada o mejor aún, un proceso de comunicación, cuyo propósito es presentar a los estudiantes de manera sistemática los hechos, ideas, técnicas y habilidades, que conforman el conocimiento humano.

Por ejemplo, en la Química, la esencia de la enseñanza se basa en el estudio de las sustancias y sus transformaciones y se explica el comportamiento de estas a partir de su estructura química, es decir, composición, tipo de partículas, ordenamiento y enlace químico que las unen, mediante la comunicación directa o apoyada en la utilización de medios de enseñanza, en especial las tecnología de la información. Tiene como objetivo lograr en los estudiantes un reflejo de la realidad objetiva en forma de conocimiento, hábitos, habilidades y convicciones que los preparen para enfrentar situaciones nuevas, de manera creadora y adaptativa. (Builes Múnera, 2015)

La Química como asignatura toma para su enseñanza un grupo de categorías de esta ciencia y las combina pertinentemente con aquellas que son ajustadas a la educación, para posibilitar la adquisición de las bases de esta ciencia y mediante ella preparar integralmente a los estudiantes para su actividad intelectual y práctica como miembros útiles de nuestra sociedad, muchos de los cuales desarrollan las distintas áreas de la química como ciencia. Así, el objeto de estudio de la asignatura Química se encamina a las sustancias y a sus transformaciones, como se ha indicado anteriormente, en función de la formación integral que la escuela, unido a la familia y a la sociedad deben lograr en los estudiantes. (García-Valcárcel Muñoz - Repiso, 2017)

La química es una ciencia teórico-experimental, por tanto, el empleo de las actividades experimentales basadas en simuladores y programas informáticos en la enseñanza, potencia la imaginación, y durante su realización los estudiantes observan, describen, comparan, establecen diferentes relaciones causales, entre ellas: la relación entre las propiedades de las sustancias, su estructura y su aplicación; entre el diseño del aparato de reacción y las propiedades de las sustancias reaccionantes y productos, que por las carencias materiales y económicas se hace difícil tener en 
Yoiler Batista Garcet, Rolando Barcia Menéndez, Raquel Vera Velàzquez...

los laboratorio, el auge de las tecnología en el siglo XXI despierta el interés constante en el aprendizaje de los estudiantes. (Autores, 2009)

\section{Metodología}

La investigación realizada se centra en la preparación de los profesores de la facultad de Ciencias de la salud en el uso de las TICs para brindar las posibilidades didácticas en la adquisición de conocimientos teóricos y prácticos de la química, aprovechando el vertiginoso desarrollo de las tecnologías en el siglo XXI.

Durante el proceso investigativo se ha utilizado, fundamentalmente, como método del nivel empírico, la observación, de carácter participante y sistemático, del proceso pedagógico que se lleva a cabo en la facultad. Para ello se ha elaborado como instrumento una guía de observación que ha permitido recolectar información sobre el empleo de las nuevas tecnologías en el desarrollo de las clases de química, lo que ha revelado la existencia del problema que se investiga.

La observación ha posibilitado valorar la pertinencia de la propuesta que se presenta como solución al problema. Dicha propuesta consiste en brindar herramientas digitales y metodológicas que permita a los docentes explotar las potencialidades de dichos medios.

\section{Enfoques pedagógicos y principios metodológicos utilizados en el proceso de enseñanza- aprendizaje de la química}

Según (Adell, 2014)los profesores más imaginativos y comprometidos ya han descubierto su potencial y han desarrollado estrategias didácticas bien fundamentadas pedagógicamente para el uso de las Tecnologías de la Información y la Comunicación en el aula y a través de éstas desarrollar de manera eficiente el Enfoque Comunicativo.

De acuerdo con el plan de estudios (Autores, 2009), la disciplina Química General pretende no solo abarcar contenidos tradicionales, sino integrar y profundizar los conocimientos que adquirieron los estudiantes en el nivel precedente, acerca de los principios, leyes y teorías más generales de la Química, y estudiar los aspectos esenciales de esta ciencia aplicados a las sustancias, las mezclas y la reacción química. La sustancia química es el tema inicial que se trata y se aborda desde el punto de vista de su composición, estructura y de la relación de esta con algunas de sus propiedades físicas. El estudio de las mezclas incide especialmente en las disoluciones acuosas; se insiste en las formas de expresar su composición y se analizan los factores energéticos involucrados en el proceso de disolución, que influyen en la solubilidad. La reacción química se analiza desde el punto de vista estructural, termodinámico, cinético y del grado de conversión que alcanza, y para ello se requiere del aprendizaje previo de las sustancias y las mezclas.

Como se ha mencionado son muchos los contenidos y son diversas las oportunidades que nos brindan las nuevas tecnologías en el correcto desarrollo del proceso docente educativo existen diversos principios que nos permiten elevar la calidad del aprendizaje en los estudiantes.

\section{Principio de interacción}

Desde varios enfoques metodológicos se destaca la importancia del contexto en el proceso de enseñanza-aprendizaje. Las tareas propuestas a los estudiantes mediante las nuevas Tecnologías deberán estar contextualizadas, ser variadas.

\section{Principio de autonomía}


Mediante los recursos propuestos el estudiante aprenderá a responsabilizarse sobre su propio aprendizaje, concepto fundamental para poder realizar tareas significativas, propuestas y mediadas por el profesor, que ayudarán al estudiante a mejorar su competencia en habilidades de formulación de los compuestos químicos. La colaboración tendrá un papel muy representativo en este proceso, fomentando la autonomía y la responsabilidad de cada individuo sobre el grupo y compartiendo el aprendizaje en dirección a un objetivo común que será la realización de la tarea.

\section{Principio de colaboración}

Implementar plenamente el trabajo colaborativo en el aula. Las tecnologías empleadas facilitarán la generación de tareas y proyectos en colaboración, permitiendo la interacción profesor-estudiante y estudiante-estudiante. Mediante los recursos se podrá utilizar las habilidades y destrezas en el desempeño de los estudiantes lo que permitirá ir adquiriendo conocimientos sólidos. Este tipo de trabajo facilitará que los estudiantes desarrollen actitudes de autonomía, liderazgo, tolerancia, comprensión, razonamiento, socialización o respeto entre muchos otros, y mejorará los procesos mentales y sociales del estudiante.

\section{Principio de motivación}

Todos los recursos aquí presentados requieren la guía del profesor, en mayor o menor medida, en su aplicación en el aula. Para todos ellos es necesario que el profesor proponga temas atractivos para los estudiantes, por los que realmente tengan interés y que les motiven para aprender a lo largo de todo el proceso. En este sentido, los recursos proporcionados, junto con los materiales auténticos que escoja el profesor (digitales o no), serán de una gran ayuda para conseguir que los estudiantes se interesen por el tema.

\section{El rol del Profesor de química y el uso de las Tecnologías de la Información y la Comunicación}

El profesor que pretende enseñar utilizando las Tecnologías de la Información y la Comunicación tiene un rol muy distinto al de un profesor tradicional. Al igual que los estudiantes, debe contar con habilidades tecnológicas mínimas, además de ser experto en el contenido de la materia. Debe ser más consciente de las necesidades del estudiante sin perder los objetivos del programa del curso. Dentro de esas habilidades tenemos, fundamentalmente, que el profesor debe ser motivador; debe ayudar al estudiante a encontrar la coherencia de las actividades que realiza en clase con el mundo real. El profesor debe influir en el estudiante para que este se comprometa, participe y persista en el logro de sus objetivos. (Carneiro, 2019)

Los profesores deben tener en cuenta que su función principal es enseñar a aprender, por tanto, debemos estar capacitados para utilizar la tecnología en el logro del aprendizaje. Así, debemos conocer las técnicas y las herramientas que sean válidas para su aplicación en clase y para la comunicación fuera de la universidad mediante el uso de medios electrónicos. Además, el rol del profesor en la sociedad del conocimiento debe ser el de garante y medio para llevar el conocimiento de las Tecnologías de la Información y la Comunicación a sus aulas.

Para (Pablos, 2012) la formación del profesorado en la era de la tecnología educativa es de gran importancia. Los profesores deben tener una formación específica, tanto en el uso técnico como pedagógico de estos modernos medios.

Gracias al acceso a las tecnologías de las comunicaciones los profesores de química en la facultada de Ciencias de la Salud de la Universidad Estatal del Sur de Manabí, podemos disponer en nuestros salones de clases de diversos recursos que nos permiten diversificar el aprendizaje ya sea presencial 
Yoiler Batista Garcet, Rolando Barcia Menéndez, Raquel Vera Velàzquez...

como virtual facilitando la comprensión de contenidos que explicándolos de una forma teórica sería muy complejo su asimilación por parte de los estudiantes. Hoy en día la información en las nuevas tecnologías va revolucionando de manera vertiginosa y los estudiantes a la par, sin embargo los docentes se van quedando rezagados en el manejo de los diversos recursos disponibles.

En la opinión de este humilde investigador los docentes deben tener;

Conocimientos sobre los procesos de comunicación y de significación de los contenidos aparecidos en los diferentes medios. Esto es educar para la información.

Conocimientos sobre las diferentes formas de trabajar las distintas tecnologías. Distintos tratamientos del conocimiento y de la información.

Conocimientos didácticos sobre las Tecnologías de la Información y la Comunicación en el aula. Estos conocimientos, al igual que las distintas Tecnologías de la Información y la Comunicación, deben ser utilizados como herramientas de manera responsable y, siempre, planificados desde las unidades didácticas y el sílabo de cada materia.

Criterios válidos para llevar a cabo la selección de materiales adecuados.

El reto que la nueva sociedad plantea a los profesores no se reduce a una actualización científicodidáctica sobre una serie de contenidos de la educación formal, ni se limita a los espacios escolares, sino que ha de tomar parte en la educación no formal de sus estudiantes, potenciando las interrelaciones entre la universidad y sociedad. El concepto de profesor ha tenido con las Tecnologías de la Información y la Comunicación un cambio profundo, por tanto, pasa de ser mero transmisor de conocimientos a guía. El estudiante también cambia, y pasa de ser un sujeto pasivo a adoptar un papel activo.

Debemos ser conscientes de todos los medios y herramientas que la sociedad del conocimiento pone a nuestra disposición. Para ello debemos estar formados y reciclarnos, y siempre entender que los nuevos recursos no son una fórmula mágica para el aprendizaje, sino un recurso más. Por eso debemos ser críticos y elegir qué medios y cuándo usarlos en nuestras clases de manera responsable y crítica.

\section{Resultados}

\section{Aspectos a considerar en la utilización de los medios en el aula de química}

Pere Marqués (Marquéz, 2015)como se ha mencionado anteriormente, cualquier actividad escogida para ser aplicada en el aula debe ser analizada y preparada para estudiantes concretos y objetivos de aprendizaje. No todas las actividades que encontramos en la red pueden ser apropiadas para ponerlas en práctica, o pueden no transmitir contenidos suficientemente significativos.

En la mayoría de los casos, los profesores, en un intento por introducir las Tecnologías de la Información y la Comunicación en el aula, se encuentran con recursos online descontextualizados, obsoletos y sin un contenido apropiado para aportar a los estudiantes. Para reducir la posibilidad de encontrarnos con que los recursos no eran tan apropiados como se creía o que no causan la reacción esperada en los estudiantes, se deberá planificar cuidadosamente nuestra intervención, analizando previamente lo significativo de cada actividad introducida.

Es conveniente que el propio profesor elabore sus propios recursos adaptados, la tecnología debe abrir la posibilidad de que sea el educador el que configure los recursos para un fin determinado. Esta recomendación cobra un sentido especial cuando de idioma inglés se trata. Para aplicar las 
Tecnologías de la Información y la Comunicación en el aula de química no sólo necesitamos tener los medios adecuados, sino que, además, necesitamos planificar un planteamiento específico de cómo pueden ajustarse a nuestro contexto para optimizar la adquisición de la lengua.

Por otra parte, será fundamental que en la preparación de actividades con recursos tecnológicos se compruebe previamente que los materiales que vamos a utilizar funcionen, simulando la tarea que deberán realizar los estudiantes y revisando con anterioridad el hardware, software y otros elementos que vayamos a necesitar. Finalmente, debemos asegurarnos y prever que los elementos organizativos sean adecuados (distribución de los estudiantes en el aula, tiempo de la actividad, etc.) y estén disponibles los espacios de la universidad donde queramos poner en práctica el recurso.

El profesor de química deberá reflexionar y plantearse una serie de interrogantes que guiarán su acción antes de la aplicación de un recurso en el aula. A continuación, se ilustran algunas de ellas que pueden servir de referencia para una acción educativa e introducción fundamentada de las Tecnologías de la Información y la Comunicación en el aula de química:

¿Cuál es la metodología que lo fundamenta?

¿Son los objetivos y contenidos lo suficientemente significativos?

¿Se ajusta al contexto disponible y a las características de los estudiantes?

¿Cumple la plataforma con los requisitos para desarrollar la clase?

¿Tienen los estudiantes suficientes contenidos y habilidades como para completar la tarea satisfactoriamente?

¿Dispongo de los suficientes elementos tecnológicos (ordenadores, Tablet, pizarra digital, etc.)?

¿Necesito algún tipo de software o programa?

¿Está presente en mi centro?

¿Qué organización es necesaria para una mejor realización de la actividad?

Selección de Tecnologías y recursos digitales para el aula

El principal punto de partida en la integración de las Tecnologías de la Información y la Comunicación debe ser la disponibilidad de equipos tecnológicos físicos debidamente instalados, ubicados y con un sistema de mantenimiento eficiente, para que de esta manera podamos utilizar los recursos digitales sin ningún tipo de impedimento, dando un sentido a los equipos. Distinguiremos dos clasificaciones: por una parte, los elementos físicos tecnológicos posibles (hardware) plataformas y por otra los recursos y medios didácticos que se pueden implementar en dichas tecnologías:

Equipos tecnológicos (hardware):

- PDI (Pizarra Digital Interactiva).

- Tablet. Teléfonos.

- Equipos informáticos portátiles y de sobremesa.

- Proyectores.

- Reproductores de CD, DVD 
- Televisión

Recursos digitales (software):

- Power Point y actividades interactivas.

- TV, videos.

- Juegos de memorización.

Otros recursos de exploración para la explotación de la química en el aula;

- Google Docs: Una herramienta perfecta para crear y compartir documentos. (http://www.youtube.com/watch)

- Issuu: para cargar y ver material digital (http://issuu.com)

- Picasa: un organizador y visor de imágenes. (http://picasa.google.com/)

- Slideshare: para compartir presentaciones y documentos. (http://www.slideshare.net)

- Blogger: página Web para crear blogs. (https://www.blogger.com/start)

- Youtube: una página para compartir vídeos. (http://www.youtube.com)

- Skype: software que permite diferentes tipos de conversaciones. (http://www.skype.com)

- JClic: aplicaciones para crear actividades educativas. (http://clic.xtec.cat/es/index.htm)

- CMapTools: programa para crear mapas conceptuales. (http://cmap.ihmc.us/download/)

- Hot Potatoes: otro sistema para crear actividades educativas. (http://hotpot.uvic.ca)

-Formularios de google para crear actividades evaluativas (https://docs.google.com/forms/u/1/?tgif=d)

-Classroom plataforma virtual para compartir materiales y modelar clases virtuales (https://classroom.google.com/u/0/h)

-Meet herramienta para impartir conferencia en grupo (https://meet.google.com/_meet?pli=1\&authuser=1)

Los profesores debemos ser un puente de unión entre las Tecnologías de la Información y la Comunicación y el aprendizaje de los estudiantes, sirviéndoles de guía en el proceso y otorgándoles un papel activo. Para ello hay que tener en cuenta dos factores primordiales:

Los profesores necesitan implicarse en su propia preparación para superar las barreras del conocimiento tecnológico.

La administración educativa debe facilitar la preparación de los profesores.

En cuanto al área de química, los profesores debemos tener claros los siguientes propósitos:

Reconocer los medios de comunicación como una herramienta que permite ver los contenidos de otra manera.

Acercar las diferentes culturas a nuestros estudiantes mediante los materiales auténticos que nos proporciona la utilización de las nuevas Tecnologías de la Información y la Comunicación.

78 UNESUM-Ciencias. Publicación cuatrimestral. Vol. 3, Año 2019, No. 2 (Mayo - Agosto) 
Desarrollar y practicar todas las habilidades por medio de los soportes audiovisuales e informáticos sin descuidar la práctica haciendo énfasis en los simuladores electrónicos.

\section{CONCLUSIONES}

La preparación permanente de los profesores constituye en la actualidad una prioridad en la educación, y en respuesta a esta necesidad, cada vez se encuentran más cursos e información para iniciar a los profesores es en la utilización de estos medios en las aulas, y promover así un aprendizaje y mejora continua a lo largo de su carrera como profesores.

No obstante, aunque la innovación es un aspecto clave en cualquier profesional implicado con su docencia, se deberá centrar la atención en que los recursos sean funcionales, operativos y fundamentalmente didácticos.

Tanto las metodologías consideradas como tradicionales, como los recursos más novedosos pueden contribuir al proceso de enseñanza-aprendizaje. El profesor necesita integrar ambos aspectos para que lleguen a complementarse sabiendo obtener provecho en cada momento de las ventajas de cada uno. Al igual que con cualquier otro enfoque o recurso, no se debe cometer el error de introducir las Tecnologías de la Información y la Comunicación en el aula como sustituto de una metodología comunicativa.

La sociedad globalizada y el constante avance de las tecnologías y la comunicación, sumado a la innovación educativa demanda de los docentes de química de la facultad de ciencias de la salud de la Universidad Estatal del Sur de Manabí un mayor esfuerzo por mantenerse en una constante actualización en el manejo de los diversos programas que la ciencia tecnológica pone a nuestro alcance. (Builes Múnera, 2015)

La utilización de la Tecnologías de la Información y la Comunicación en la docencia, permite que los estudiantes posean las herramientas necesarias que les permitan actuar en el nuevo contexto universitario con mayor independencia, lo que se traduce en una mayor calidad del futuro egresado de laboratorio Clínico.

\section{REFERÉNCIAS BIBLIOGRÁFICAS}

Adell, J. (2014). Internet en el aula. Obtenido de http://www.cyta.com.ar/alearn/wq/wq_archivos/AdellWQ.pdf.

Autores, C. d. (2009). Plan de estudios "D" carrera de Licenciatura en Química. Ministerio de Educación Superior. La Habana: Editorial Pueblo y Educación.

Builes Múnera, R. A. (2015). El aporte de computadores para educar en la implementación de la enseñanza innovadora. Revista virtual Universidad Católica del Norte, 44.

Cardozo, J.C.G, Navarro, S.M.B \& Álvarez, D.J:S. (2017). Diseño e implementación de recursos educativos digitales abiertos inclusivos: en el marco de la estrategia de formación y acceso de computadores para educar . Revista $Q, 9$ (18).

Carneiro, R. (2019). Los desafios de las TIC para el cambio educativo. España: Fundación Santillana.

García-Valcárcel Muñoz - Repiso, A. M.-P. (2017). Elaboración y validación de rúbrica para evaluación de Trabajos de fin de Máster (TFM) pertenecientes al Máster "Las TIC en educación, Análisis y diseño de procesos, recursos y prácticas formativas. España: Universidad Salamanca.

Gónzález,E. (2019). La era de las Nuevas Tecnologías. En E. González. La Habana: Pablo de la Torriente Brau.

Marquéz, P. (2015). Los Medios Didácticos. Obtenido de facultad de educación. Obtenido de http://peremarques.pangea.org/medios.htm. 
Yoiler Batista Garcet, Rolando Barcia Menéndez, Raquel Vera Velàzquez...

Pablos, P. J. (2012). Tecnología Educativa: La formación del profesorado en la era de Internet. . Malaga, España: Ijibe.

80 UNESUM-Ciencias. Publicación cuatrimestral. Vol. 3, Año 2019, No. 2 (Mayo - Agosto) 